\title{
Exploratory Factor Analysis and Confirmatory Factor Analysis of the Korean Version of Hypomania Checklist-32
}

\author{
Doyoun An, Kyung Sue Hong and Ji-Hae Kim ${ }^{凶}$ \\ Department of Psychiatry, Samsung Medical Center, Sungkyunkwan University, School of Medicine, Seoul, Korea
}

\begin{abstract}
Objective The Hypomania Checklist - 32 (HCL-32) is a self-assessment instrument developed by Angst et al. (2005) to identify bipolarity in the general population, as well as patients with unipolar depression. The principal objective of this study was to assess the factor structure of the Korean version of the HCL-32 for mood disorder patients, via exploratory factor analysis (EFA) and confirmatory factor analysis (CFA).

Methods The Korean version of HCL-32 were evaluated in a sample of 608 mood disorder patients, including 210 bipolar disorder patients and 398 unipolar disorder patients, all of whom were diagnosed in accordance with either the Structured Clinical Interview of DSM-IV or Mini International Neuropsychiatric Interview They were divided randomly into two groups, and then the EFA was administered to group 1 and the CFA was administered to group 2.

Results A 3-factor structure for the HCL-32 was generated, which explained $44 \%$ of the total variance from EFA. Factor 1 , comprising 18 items, was designated as 'elated mood/increased energy'; factor 2, comprising 8 items, was designated as 'risk-taking behavior/irritability'; and factor 3, comprising 2 items, was designated as 'increased sexual activity'. Researchers confirmed the 3-factor solution derived from group 1 by the CFA.

Conclusion The primary findings of this study were the replication and confirmation of the 3-factor structure in Korean mood disorder patients; our results were consistent with previous EFAs.

Psychiatry Investig 2011;8:334-339
\end{abstract}

Key Words Hypomania Checklist-32 (HCL-32), Factor analysis, Bipolar Disorder.

\section{INTRODUCTION}

Major depressive disorder (MDD) is characterized by one or more major depressive episodes, and bipolar disorder (BP) is characterized by one or more manic, mixed, or hypomanic episodes, frequently coupled with one or more major depressive episodes. Thus, individuals undergoing depressive episodes are diagnosed as with/without past manic or hypomanic episodes [Diagnostic and Statistical Manual of Mental Disorder, Fourth Edition Text Revision (DSM-IV-TR) ${ }^{1}$ ]. However, bipolar II disorder (BP II) and bipolar disorder not otherwise specified (BP NOS) are frequently underdiagnosed or misdiagnosed as MDD, as the hypomania in these cases is charac-

Received: November 24, 2010 Revised: May 12, 2011

Accepted: May 18, 2011 Available online: December 8, 2011

$\triangle$ Correspondence: Ji-Hae Kim, PhD

Department of Psychiatry, Samsung Medical Center, Sungkyunkwan University, School of Medicine, 50 Ilwon-dong, Kangnam-gu, Seoul 135-710, Korea Tel: +82-2-3410-0931, Fax: +82-2-3410-0050, E-mail: jihae0931.kim@samsung.com

(c) This is an Open Access article distributed under the terms of the Creative Commons Attribution Non-Commercial License (http://creativecommons.org/licenses/bync/3.0) which permits unrestricted non-commercial use, distribution, and reproduction in any medium, provided the original work is properly cited. terized by lower levels of dysfunction and does not require hospitalization, as compared with the mania. ${ }^{2-5}$ It often requires 8-10 years to properly diagnose and treat BP patients ${ }^{3,6,7}$ and the relevant occupational/interpersonal impairments and suicidal risk generally increase over that time. ${ }^{8,9}$ Therefore, appropriate diagnosis and treatment can be achieved via careful inquiry into past (hypo)manic episodes.

Angst et al. ${ }^{3}$ previously devised the Hypomania Checklist-32 (HCL-32), the effective self-assessment screening instrument for the detection of hypomania from unipolar MDD. Recently, a variety of studies have been conducted to validate and characterize the factor structure and its characteristics in Spain, Italy, German, Taiwan, and Korea. Angst et al. ${ }^{3}$ proposed a 2-factor solution of "active/elated" and "risk-taking/irritable", to be determined via exploratory factor analysis (EFA). The possibility exists that the active/elated factor--which consists of overactivity, mood elation, and improved thinking-involves less pathological symptoms, and the risk-taking/irritable factor-which consists of risk-taking behavior, anger/irritability, and flight of ideas--is more profoundly related to diverse dysfunction and target features of treatment. ${ }^{3,10}$ Meyer et al. ${ }^{10}$ pre- 
viously identified the risk-taking/irritable factor as the "dark side of hypomania", which they associated with the impairments associated with the hypomania group. Additionally, the results of a study conducted in Taiwan are generally supportive of the 2-factor solution. ${ }^{11}$ Similarly, the EFA for the Korean version of HCL-32 also yielded a 2-factor solution--"elated mood/increased energy" and "irritability/ impulsivity".12

The Polish study conducted to determine the utility of HCL32 in discriminating between patients with treatment-resistant and treatment non-resistant depression proposed a 3-factor solution, consisting of "elevated mood/increased activity", "sexual activity", and "irritability". ${ }^{13}$ Holtman et al. ${ }^{14}$ tested the psychometric properties on the HCL-32 in a sample of nonclinical adolescents, and produced a 3 -factor structure consisting of "active-elated", "disinhibited/stimulation-seeking", and "irritable-erratic"; the "active-elated" factor was regarded as "sunny, bright" hypomania, and the "disinhibited/stimulation-seeking" and "irritable-erratic" factors were related to the "dark" expression of bipolarity.

The principal objective of this study was to determine the proper factor solution for Korean mood disorder patients via EFA and confirmatory factor analysis (CFA) of HCL-32.

\section{METHODS}

\section{Patients}

Mood disorder patients who were diagnosed with bipolar disorder (BP I, BP II, BP NOS) or unipolar disorder [MDD, depressive disorder not otherwise specified (DEP NOS), dysthymic disorder (DD)] via Structured Clinical Interview of DSM-IV or Mini International Neuropsychiatric Interview screening interviews were recruited from the Department of Psychiatry at the Samsung Medical Center. A total of 608 patients were enrolled; 410 (67.4\%) were female. The sample included 262 inpatients and 346 outpatients. The mean age of the patients was $42.6 \pm 15.7$ years (range 18-84). Among these patients, 210 were classified as bipolar disorder and 398 as unipolar disorder. In the bipolar disorder group, BP I consisted of 78 patients, BP II consisted of 88 patients, and BP NOS consisted of 44 patients. The unipolar disorder group included $264 \mathrm{MDD}$ patients, 97 DEP NOS patients, and $37 \mathrm{DD}$ patients. Table 1 shows the sample's sociodemographics, clinical characteristics, and total HCL-32 scores.

Among the patients, 100 (16.4\%) had one or more comorbid Axis I disorders based on the DSM-IV-TR: 87 patients had two disorders, 12 patients had three disorders, and 1 patient had four Axis I disorders. Upon secondary diagnosis, $54 \mathrm{pa}-$ tients were found to have anxiety disorders, 20 patients had other mood disorders, 15 patients had substance-related disorders, 8 patients had eating disorders, and 3 patients had so- matoform disorders.

Exclusion criteria were intensity of depression $\geq 20$ on the 17-item Hamilton Depression Rating Scale (HDRS), or intensity of mania $\geq 15$ on the Young Mania Rating Scale (YMRS), which could influence the recall of hypomanic symptoms in the past, known histories of schizophrenia or other psychotic disorders, neurological disorders, and mental retardation.

Written informed consent was obtained from all patients, based on complete information regarding this study; all protocols of this study were approved by the Institutional Review Board of the Samsung Medical Center.

\section{Measures}

\section{Korean version of the Hypomania Checklist-32}

HCL-32 is a self-assessment instrument comprising 32 item$\mathrm{s}$ for detecting hypomanic symptoms, as initially developed by Angst et al. ${ }^{3}$ The individuals were instructed to answer yes/no questions about emotions, thoughts, or behaviors, including items such as: "My mood is higher, more optimistic", "I think faster", "I need less sleep" or "I get into more quarrels" in 'a period when you were in a "high" state. The instrument also includes questions regarding the duration, the impact of family, social and work life, or people's reactions such as "positive and negative", "positive", "no impact" or "negative". We employed the Korean version of the HCL-32, as validated by $\mathrm{Oh}$ et al. ${ }^{12}$, and the internal consistency was suffcient, with an Cronbach's alpha $=0.89$ in this study.

\section{Statistics}

Before conducting factor analysis, patients were randomly divided into two groups. The Independent Sample t-test and the Chi-square test were used to determine the differences in demographic variables and the clinical characteristics between groups. Upon comparison of group characteristics between groups 1 and 2, no significant differences in age, gender, education year, diagnosis-groups, HCL-32 score, YMRS score and HDRS scores were noted to exist between groups.

Researchers conducted EFA for group 1 using a tetrachoric correlation matrix calculated for the 32 dichotomous items and an oblique factor rotation (geomin). Factors were selected if their eigenvalue was $>1$ and the number of factors was determined according to the results of a Scree test, as well as the coherence and interpretability of the factors. Items allocated to a specific factor were based on a loading of $\geq 0.30$ on that factor, and items for which the difference of factor loadings was less than 0.10 were excluded.

Researchers conducted CFA for group 2, and 3 types of fit indices were employed to evaluate the models: Comparative Fit Index (CFI), Root-Mean Square Error of Approximation 
Table 1. Demographic data, clinical characteristics and HCL-32 scale score by diagnostic groups

\begin{tabular}{|c|c|c|c|c|c|c|c|c|c|}
\hline & $\begin{array}{c}\text { Total } \\
\mathrm{N}=608\end{array}$ & $\begin{array}{c}\text { BP I } \\
\mathrm{N}=78\end{array}$ & $\begin{array}{c}\text { BP II } \\
\mathrm{N}=88\end{array}$ & $\begin{array}{c}\text { BP NOS } \\
\mathrm{N}=44\end{array}$ & $\begin{array}{c}\mathrm{MDD} \\
\mathrm{N}=264\end{array}$ & $\begin{array}{c}\text { DEP NOS } \\
\mathrm{N}=97\end{array}$ & $\begin{array}{c}\mathrm{DD} \\
\mathrm{N}=37\end{array}$ & $\mathrm{p}$ & Post-hoc \\
\hline \multicolumn{10}{|l|}{ Age } \\
\hline Mean \pm SD & $42.6 \pm 15.7$ & $35.6 \pm 12.3$ & $34.5 \pm 12.9$ & $34.0 \pm 14.7$ & $45.6 \pm 15.3$ & $50.0 \pm 14.8$ & $45.3 \pm 16.1$ & $0.000^{*}$ & \\
\hline \multicolumn{10}{|l|}{ Gender (\%) } \\
\hline Male & $198(32.6)$ & $26(33.3)$ & $26(29.5)$ & $17(38.6)$ & $92(34.8)$ & $27(27.8)$ & $10(27.0)$ & $0.660^{\dagger}$ & \\
\hline Female & $410(67.4)$ & $52(66.7)$ & $62(70.5)$ & 27 (61.4) & $172(65.2)$ & $70(72.2)$ & $27(73.0)$ & & \\
\hline \multicolumn{10}{|c|}{ Education (\%) } \\
\hline 0 & 8 & 0 & 0 & 2 & 4 & 2 & 0 & & \\
\hline $1-6$ & 53 & 2 & 2 & 0 & 31 & 13 & 5 & $0.003^{\dagger}$ & \\
\hline $7-12$ & 247 & 29 & 35 & 26 & 98 & 42 & 17 & & \\
\hline $13-$ & 300 & 47 & 51 & 16 & 131 & 40 & 157 & & \\
\hline HCL-32 & $12.6 \pm 7.2$ & $15.4 \pm 7.0$ & $18.1 \pm 6.2$ & $16.6 \pm 5.5$ & $11.4 \pm 6.7$ & $7.7 \pm 5.6$ & $10.7 \pm 5.6$ & $0.000^{*}$ & $\begin{array}{l}\mathrm{BPI}=\mathrm{BPII}=\mathrm{BP} \\
\mathrm{NOS}>\mathrm{MDD}=\mathrm{DD}, \mathrm{DEP} \\
\text { NOS }\end{array}$ \\
\hline
\end{tabular}

*ANOVA, ${ }^{\dagger}$ chi-square test, post-hoc: Bonferroni-Holm correction, BP I: bipolar I disorder, BP II: bipolar II disorder, BP NOS: bipolar disorder not otherwise specified, MDD: major depressive disorder, DEP NOS: depressive disorder not otherwise specified, DD: dysthymic disorder, HCL-32: Hypomania Checklist-32, SD: standard deviation

(RMSEA), and chi-squared tests. In this study, an acceptable model fit was defined as follows: CFI $\geq 0.90$ and RMSEA $\leq 0.08$. The chi-squared tests are evaluated in two ways. First, if the results of chi-squared tests are not significant, the model does not deviate from the data. Second, if the chi-squared statistics are significant but less than twice the degrees of freedom, the model is regarded as a good representation of the data.

All computations were conducted using SPSS 17.0 (SPSS, Inc., 2008), MPlus2.14 (Muthen \& Muthen, 1998), and CEFA 3.02 software (Browne, Cudeck, Tateneni, \& Mels, 2008).

\section{RESULTS}

\section{Exploratory factor analysis}

The results of EFA revealed seven factors with eigenvalues of $>1$, but the three factors were selected by Scree test, along with the coherence and interpretability of the factors (Table 2). Among the 32 items, 4 items were excluded: item 29 (I drink more coffee), item 30 (I smoke more cigarettes), item 31 (I drink more alcohol), and item 32 (I take more drugs). Items 29, 30, and 32 were excluded due to the fact that their factor loadings were below 0.30 . Item 31 had cross-loading on factor 2 (0.362) and factor 3 (0.380), and was therefore excluded.

The 3 -factor solution yielded and explained $44.0 \%$ of the total variance. Factor 1 consisted of 18 items ('I need less sleep', 'I feel more energetic and more active ', 'I am more self-confident', 'I enjoy my work more, 'I am more sociable [make more phone calls, go out more]', 'I want to travel and do travel more', I am physically more active [sports, etc]', 'I plan more activities or projects', 'I have more ideas, I am more creative' , 'I am less shy or inhibited' , 'I wear more colorful and more extravagant clothes/makeup', 'I want to meet or actually do meet more people', 'I talk more,' 'I think faster,' 'I make more jokes or puns when I am talking,' 'I engage in lots of new things,' I do things more quickly and/or more easily', 'My mood is higher, more optimistic') and accounted for $28.2 \%$ of the total variance. This factor was labeled 'elated mood/increased energy'.

Factor 2 included 8 items ['I tend to drive faster or take more risks when driving,' 'I spend more/too much money', 'I take more risks in my daily life (in my work and/or other activities)', 'I am more easily distracted', 'My thoughts jump from topic to topic', 'I am more impatient and/or get irritable more easily', 'I can be exhausting or irritating for others', 'I get into more quarrels'] and accounted for $10.9 \%$ of the total variance. We named this factor 'risk-taking behavior/irritability'.

Factor 3 included 2 items ('I am more interested in sex and/ or have increased sexual desire,' 'I am more flirtatious and/or am more sexually active') and accounted for $4.9 \%$ of the total variance. We labeled this factor 'increased sexual activity'.

The correlation of factor 1 and factor 2 was measured to be 0.243 , the correlation of factor 1 and factor 3 was 0.323 , and the correlation of factor 2 and factor 3 was 0.147 .

\section{Confirmatory factor analysis}

On the basis of earlier empirical studies, we compared four factor models. For Model 1, researchers considered the 2-factor model including 26 items as suggested by Angst et al. ${ }^{3}$ on the study of development of the HCL-32. The two factors were: "activated/elated" and "risk-taking/irritable". Model 2 
Table 2. Item loadings for the three factors of the Hypomania Checklist-32 from the exploratory factor analysis

\begin{tabular}{|c|c|c|c|}
\hline \multirow{2}{*}{ Items } & \multicolumn{3}{|c|}{ Factor } \\
\hline & F1 & $\mathrm{F} 2$ & F3 \\
\hline 1. I need less sleep. & 0.338 & & \\
\hline 2. I feel more energetic and more active. & 0.804 & & \\
\hline 3. I am more self-confident. & 0.845 & & \\
\hline 4. I enjoy my work more. & 0.829 & & \\
\hline 5. I am more sociable (make more phone calls, go out more). & 0.656 & & \\
\hline 6. I want to travel and do travel more. & 0.420 & & \\
\hline 7. I tend to drive faster or take more risks when driving. & & 0.423 & \\
\hline 8. I spend more/too much money. & & 0.416 & \\
\hline 9. I take more risks in my daily life (in my work and/or other activities). & & 0.482 & \\
\hline 10. I am physically more active (sports, etc). & 0.357 & & \\
\hline 11. I plan more activities or projects. & 0.744 & & \\
\hline 12. I have more ideas, I am more creative. & 0.730 & & \\
\hline 13. I am less shy or inhibited. & 0.596 & & \\
\hline 14. I wear more colorful and more extravagant clothes/makeup. & 0.442 & & \\
\hline 15. I want to meet or actually do meet more people. & 0.674 & & \\
\hline 16. I am more interested in sex and/or have increased sexual desire. & & & 0.712 \\
\hline 17. I am more flirtatious and/or am more sexually active. & & & 0.726 \\
\hline 18. I talk more. & 0.652 & & \\
\hline 19. I think faster. & 0.786 & & \\
\hline 20. I make more jokes or puns when I am talking. & 0.522 & & \\
\hline 21. I am more easily distracted. & & 0.593 & \\
\hline 22. I engage in lots of new things. & 0.673 & & \\
\hline 23. My thoughts jump from topic to topic. & & 0.456 & \\
\hline 24. I do things more quickly and/or more easily. & 0.700 & & \\
\hline 25. I am more impatient and/or get irritable more easily. & & 0.670 & \\
\hline 26. I can be exhausting or irritating for others. & & 0.521 & \\
\hline 27. I get into more quarrels. & & 0.376 & \\
\hline 28. My mood is higher, more optimistic. & 0.708 & & \\
\hline \multicolumn{4}{|l|}{ 29. I drink more coffee. } \\
\hline \multicolumn{4}{|l|}{ 30. I smoke more cigarettes. } \\
\hline \multicolumn{4}{|l|}{ 31. I drink more alcohol. } \\
\hline 32. I take more drugs (sedatives, anxiolytics, stimulants). & & & \\
\hline
\end{tabular}

used 18 items, and was also a 2-factor model (identified by Wu et al. $)^{11}$ used in Taiwan-a country with an oriental cultural background similar to that of Korea. Model 3 was a 3-factor model for 15 items suggested by Rybakowsky et al. ${ }^{13}$ : "elevated mood/increased activity", "sexual activity", and "irritability". Model 4 was our 3-factor model which we derived using the EFA on Group 1. In the study conducted in Korea by Oh et al. ${ }^{12}$ the 2-factor model was also generated. However, we did not employ that model in our study, since Oh's factor structure was extracted from a sample of 200 patients; this sample was far too small for a proper identification of factor structure.

Table 3 shows the goodness-of-fit statistics for the hypothesized models. In all models, CFI fulfilled the established guidelines for adequate fit. Model 1 demonstrated an inadequate RMSEA fit to the observed data. The CFA statistics for Model 2 and 4 were better than those of Model 3 and the chisquared statistics and RMSEA of Model 4 were relatively better than the results of Model 2. Thus, overall, Model 4 affords a reasonable description of the data. The following values were obtained: $\chi_{93}^{2}=213.6$ ( $\mathrm{p}<0.0001$ ), CFI=0.946, RMS-EA= 0.073 . 
Table 3. Confirmatory factor analyses of the Hypomania Checklist-32

\begin{tabular}{|c|c|c|c|c|c|}
\hline & \multicolumn{3}{|c|}{ Goodness of fit indices } & \multirow{2}{*}{ CFI } & \multirow{2}{*}{ RMSEA } \\
\hline & $\chi^{2}$ & $\mathrm{df}$ & $\chi^{2} / \mathrm{df}$ & & \\
\hline 1. Angst's (2005) 2-factor model & 233.2 & 84 & 2.77 & 0.933 & 0.085 \\
\hline 2. Wu's (2007) 2-factor model & 132.6 & 57 & 2.32 & 0.965 & 0.075 \\
\hline 3. Rybakowsky’s (2009) 3-factor model & 107.7 & 44 & 2.44 & 0.969 & 0.077 \\
\hline 4. Research 3-factor model & 213.6 & 93 & 2.29 & 0.946 & 0.073 \\
\hline
\end{tabular}

CFI: Comparative Fit Index, RMSEA: Root-Mean Square Error of Approximation

\section{DISCUSSION}

The principal objective of this study was to identify the factor structure of the Korean Version of HCL-32, via EFA and CFA analyses. The three-factor structure of HCL-32 was replicated in Korean patients with mood disorders, consistent with earlier EFA results, ${ }^{13,14}$ though the items included differed among each of the studies, owing to differences in the factor loading criteria among studies, such as $>0.30$ or $>0.40$. In our study, factor 1 was 'elated mood/increased energy' and included 18 items $(1,2,3,4,5,6,10,11,12,13,15,18,19$, $20,22,24,28)$, factor 2 was 'risk-taking behavior/irritability' and included 8 items $(7,8,9,21,23,25,26,27)$, and factor 3 was 'increased sexual activity' and included 2 items $(16,17)$. We segregated factors associated with sexual activity with items 16 and 17 ('I am more interested in sex and/or have increased sexual desire' and 'I am more flirtatious and/or am more sexually active').

Rybakowsky et al. ${ }^{13}$ also suggested a three-factor solution comprising 15 items: "elevated mood/increased activity" (items 2,3,4,5,11,12,15,20,24,28), "sexual activity" (items 16, 17), and "irritability" (items 25,26,27). ${ }^{13}$ The excluded items in the Polish study were risk-taking behaviors or distractibilities ('I tend to drive faster or take more risks when driving', 'I take more risks in my daily life', 'I am more easily distracted,' 'I think faster,' 'I engage in lots of new things', etc.) unlike our three-factor model. The factor structures of the HCL-32 with mood disorder patients included both bipolar disorder and unipolar disorder might differ somewhat from those of the unipolar depression group, allowing that the factor structure was extracted from only the unipolar depression patients in the Polish study.

Angst described a two-factor solution composed of an activated/elated factor (items 2,3,4,5,6,10,11,12,13,15,16,18,19, $20,22,24,28$ ) and a risk-taking/irritable factor (items 7,8,9,21, $24,25,26,27,31) .{ }^{3}$ Wu's research also yielded a two-factor structure: factor 1 included items 2,3,4,5,10,11,12,13,15,19,20 ,22, and 24 and factor 2 included items 7,21,25,26, and 27.11 As compared with the results of previous studies, the items in the 'increased sexual activity' factor were excluded in the 2 -factor solution. Apart from the separate factor for sexual ac- tivity obtained in our study, our results are generally in agreement with those of previous studies showing the 2-factor solution representing "activated/elated" and "risk-taking/irritable" factors, which reflect the sunny, positive side and the dark, negative side of hypomania. . $^{3,10,11,13}$

Additionally, items 29, 30, 31, and 32 ['I drink more coffee', 'I smoke more cigarettes', 'I drink more alcohol', and 'I take more drugs (sedatives, anxiolytics, stimulants)'] related to stimulation-seeking behaviors were eliminated owing to factor loadings below 0.30 or 0.40 in most factor models. This result may be due to the exclusion of patients with comorbid substance abuse or dependence from the Taiwanese and Polish studies ${ }^{11,13}$ and the low percentage of positive responses on the four items $(29,30,31,32)$ in our studies. Nevertheless, stimulation-seeking behaviors such as increased coffee or alcohol consumption were important features of hypomania, considering that the BP patients reported significantly more positive responses than the MDD or non-BP patients. ${ }^{3,14}$

The CFA results support our research model (3-factor solution) as the most reasonable fit for explaining the factor structure of hypomania symptoms in Korean patients with mood disorders. As mentioned above, our results generally agree with those of previous studies showing the 2-factor solution representing "activated/elated" and "risk-taking/irritable" factors which reflect the sunny, positive side and the dark, negative side of hypomania ${ }^{3,10,11,13}$, apart from the separate factor for sexual activity obtained in our study.

Several limitations must be taken into account when interpreting our results. First, the CFA results evidenced relatively small differences of fit indices among models. Thus, these findings require replication to robustly confirm the factor structure of hypomania symptoms in Korean mood disorder patients. Furthermore, we nevertheless segregated increased sexual activity factors, and did not clarify the psychometric properties of the three factors. The psychometric properties of three factors should be examined in future studies.

To the best of our knowledge, despite these weaknesses, this study is the first to confirm the factor structure of HCL32 for Korean mood disorder patients. In particular, recall bias was controlled to some degree by enrolling patients with only remission or subsyndromal symptoms; by so doing, we 
achieved valid results for the Korean version of the HCL-32. The results of this study may also prove helpful in reducing the HCL-32 to a short version for more effectively and quickly differentiating (hypo)mania from unipolar disorder.

\section{REFERENCES}

1. American Psychiatric Association. Diagnostic and Statistical Manual of Mental Disorders. Fourth Edition. Test Revision. Washington, DC: American Psychiatric Association; 2000.

2. Hantouche EG, Akiskal HS, Lancrenon S, Allilaire JF, Sechter D, Azorin JM, et al. Systematic clinical methodology for validating bipolar-II disorder: data in mid-stream from a French national multi-site study (EPIDEP). J Affect Disord 1998;50:163-173.

3. Angst J, Adlofsson R, Benazzi F, Gamma A, Hantouche E, Meyer TD, et al. The HCL-32: towards a self-assessment tool for hypomanic symptoms in outpatients. J Affect Disord 2005;88:217-233.

4. Bhargava Raman RP, Sheshadri SP, Janardhan Reddy YC, Girimaji SC, Srinath S, Raghunandan VN. Is bipolar II disorder misdiagnosed as major depressive disorder in children? J Affect Disord 2007;98:263-266.

5. Muzina DJ, Kemp DE, McIntyre RS. Differentiating bipolar disorders from major depressive disorders: treatment implications. Ann Clin Psychiatry 2007;19:305-312

6. Hirschfeld RM, Lewis L, Vornik LA. Perceptions and impact of bipolar disorder: how far have we really come? Results of the national depressive and manic-depressive association 2000 survey of individuals with bipolar disorder. J Clin Psychiatry 2003;64:161-174.

7. Ghaemi SN, Sachs GS, Chiou AM, Pandurangi AK, Goodwin K. Is bipolar disorder still underdiagnosed? Are antidepressants overutilized? J Affect Disord 1999;52:135-144.

8. Coryell W, Scheftner W, Keller M, Endicott J, Maser J, Klerman GL. The enduring psychosocial consequences of mania and depression. Am J Psychiatry 1993;150:720-727.

9. Gamma A, Angst J, Ajdacic-Gross V, Rössler W. Are hypomanics the happier normals? J Affect Disord 2008;111:235-243.

10. Meyer TD, Hammelstein P, Nilsson LG, Skeppar P, Adolfsson R, Angst J. The Hypomania Checklist (HCL-32): its factorial structure and association to indices of impairment in German and Swedish nonclinical samples. Compr Psychiatry 2007;48:79-87.

11. Wu YS, Angst J, Ou CS, Chen HC, Lu RB. Validation of the Chinese version of the Hypomania Checklist (HCL-32) as an instrument for detecting hypo(mania) in patients with mood disorders. J Affect Disord 2008; 106:133-143.

12. Oh MY, Angst J, Sung TH, Lee EH, Hong KS, Lee DS, et al. Reliability and validity of the Hypomania Symptom Checklist-32 in Korea. Korean J Clin Psychol 2009;28:321-338.

13. Rybakowski JK, Angst J, Dudek D, Pawlowski T, Lojko D, Siwek M, et al. Polish version of the Hypomania Checklist (HCL-32) scale: the results in treatment-resistant depression. Eur Arch Psychiatry Clin Neurosci 2010;260:139-144.

14. Holtmann M, Pörtner F, Duketis E, Flechtner HH, Angst J, Lehmkuhl G. Validation of the Hypomanic Checklist (HCL-32) in a nonclinical sample of German adolescents. J Adolesc 2009;32:1075-1088. 